\title{
Application of Computer-Aided Design (CAD) and Three-Dimensional (3D) Visualization Technologies in the Diagnosis and Treatment of Refractory Thyroid Tumors
}

This article was published in the following Dove Press journal:

Cancer Management and Research

\author{
Yi-Bo Chen \\ Zhao-Hui Wang \\ Gui-Ming Fu (DD \\ Quan-Xin Wan \\ Xiao-Jing Li \\ Jin Chen
}

Department of Head and Neck Oncology, Sichuan Cancer Hospital, Chengdu 61004I, People's Republic of China
Correspondence: Zhao-Hui Wang Email wangzh912@163.com
Background: To evaluate the application of computer-aided design (CAD) and three-dimensional (3D) visualization techniques in the diagnosis and treatment of refractory thyroid tumors.

Materials and Methods: The clinical data from 12 cases of refractory thyroid tumors treated with CAD and 3D visualization techniques from September 2016 to January 2018 were analyzed retrospectively. Ten cases were malignant, while two cases were benign. All tracheas in the 12 cases were invaded or oppressed by the thyroid tumors. Six of the cases experienced type III dyspnea, while the other six cases had type II dyspnea. All patients underwent contrast-enhanced computed tomography (CT) imaging.

Results: $\mathrm{CAD}$ and 3D visualization technologies were used to guide the surgeries. Ten cases of malignant thyroid tumors underwent total thyroidectomy, cervical lymph node dissection, and invaded organ resection. Among the ten cases, five underwent sleeve resections and anastomosis reconstructions, one underwent a tracheal sleeve resection and total laryngectomy, two underwent tracheal window resections, one case underwent a sternotomy, and one case underwent a laryngopharyngectomy, invaded skin resection, and pectoralis major muscle flap reconstruction. The two cases of benign thyroid tumors underwent subtotal thyroidectomies. Using CAD and 3D visualization techniques, surgeons can visually observe the relationship of the tumor with the respiratory tract and essential blood vessels, which can lead to optimized surgical plans and improved surgical outcomes.

Conclusion: CAD and 3D visualization technologies have an important role in the personalized surgical intervention of thyroid cancer, which will likely have important clinical implications.

Keywords: refractory thyroid tumors, thyroid cancer, computer-aided design, CAD, 3D visualization, contrast-enhanced $\mathrm{CT}$, thyroidectomy

\section{Introduction}

Thyroid cancer is the most commonly diagnosed endocrine malignancy worldwide. ${ }^{1}$ Currently, thyroid cancer accounts for $3.1 \%$ of all new cancer cases, and women are 3-times more likely to develop thyroid cancer than men. ${ }^{2}$ Surgical intervention is the first-line treatment for most patients with thyroid malignancies and some patients with benign thyroid nodules. ${ }^{3}$ Due to the anatomical location of the thyroid, locally advanced thyroid carcinomas are likely to invade the surrounding tissues, such as the larynx, trachea, recurrent laryngeal nerve, pharynx, and 
esophagus, which increases the risk of surgical complications. ${ }^{4}$ Large benign thyroid tumors, such as retrosternal goiter, which extend deep into the mediastinum, also present with a high risk of surgical complications. Due to the complexity and high risk associated with surgical intervention, the accurate and timely preoperative assessment of patients is critical to ensure optimal patient outcomes.

While traditional imaging technologies, such as contrast-enhanced computed tomography (CT) and magnetic resonance imaging (MRI), can help physicians understand the connection between the tumor and its surrounding tissues, these imaging technologies are often limited to a single section. For surgical intervention, physicians must establish three-dimensional (3D) images through spatial thinking based on two-dimensional images. Computer-aided design (CAD) and 3D visualization technologies utilize the raw data from $\mathrm{CT}$, MRI, positron emission tomography (PET), and other modalities, to construct detailed and comprehensive 3D models that can aid physicians during surgery. The 3D model provides detailed information about the lesion and the surrounding tissues in the surgical area, which can aid in disease assessment, surgical planning, intraoperative navigation, and the postoperative evaluation of patients. ${ }^{5,6}$ The CAD technology is widely used in the fields of maxillofacial surgery, plastic and reconstructive surgery, hepatic surgery, and robotic surgery, yet it has not been reported for thyroid tumors.

From November 2016 to January 2018, 12 cases of refractory thyroid cancer were successfully treated with surgery in the Head and Neck Oncology Center using CAD and $3 \mathrm{D}$ visualization technologies. In this study, we aimed to demonstrate the effectiveness of CAD with $3 \mathrm{D}$ visualization technologies for imaging refractory thyroid tumors.

\section{Materials and Methods}

\section{Demographics and Clinical Data of}

\section{Patients}

This study protocol was approved by the Ethics Committee of Sichuan Cancer Hospital (Approval Date: September 27, 2019). All procedures performed in studies involving human participants were in accordance with the ethical standards of the institutional and national research committee and with the 1964 Helsinki Declaration and its later amendments. Informed consent was waived because of the retrospective nature of the study, and the analysis used anonymous clinical data. For the case reports, written, informed consent for the publication of all case details and any images was obtained from all individual participants involved in the study. A retrospective analysis of 12 patients was performed, of which seven cases were female and five were male. The ages ranged from 46 to 74 years, with an average of 53.2 years. This was the initial diagnosis of seven cases, while five cases had recurrent disease. All 12 patients suffered from substernal goiter or T4 thyroid carcinoma. Only patients with operable tumors were considered for this retrospective study. Two cases had benign lesions, while the remaining ten cases had a malignant disease with 9 of the ten cases having trachea invasion. $^{7}$

\section{Data Procurement and CAD}

All patients underwent preoperative computed tomography (CT) and computed tomography angiography (CTA) prior to surgery using the Philips Brilliance 256-layer Spiral CT system. The scanning parameters included: voltage $120 \mathrm{kV}$, current $120 \mathrm{~mA}$, matrix $512 \times 512$, layer thickness $0.625 \mathrm{~mm}$, threshold $150 \mathrm{HU}$, arterial scan delay 10-20 s; and included plain scan images, arterial phase images, and venous phase images. Next, the DICOM formatted imaging data were transmitted to the 3D-Matic (M3D) digital medical software (Anhui King Star Digital S\&T Co. Ltd) and processed in the M3D software. Tumor lesions were converted into threedimensional (3D) images. Under the guidance of 3D images, combined with the preoperative endoscopic examination and other imaging examinations, the surgeon assessed the size of the lesion and the involved structures, and planned the surgical approach, the scope of resection, and the reconstruction options.

\section{Surgical Procedures}

The $\mathrm{CAD}$ and $3 \mathrm{D}$ visualization images were used to determine the location and extent of the tumor for surgical purposes. Two patients with giant retrosternal goiter underwent subtotal thyroidectomy, and all tumors were successfully excised through a neck approach. Ten patients with thyroid malignancies underwent thyroidectomy, cervical lymph node dissection, resection, and reconstruction of the invaded organs. The surgical procedures of the invaded organs were as follows: five cases of end-to-end anastomosis with tracheal sleeve resection, one case of total laryngectomy and tracheostomy, two cases of window or wedge resection and tracheostomy, one case of sternal dissection and mediastinum tracheal resection of low tracheostomy, two cases of pectoralis major myocutaneous flap to repair hypopharyngeal and 
skin defects. Two cases of grade III dyspnea were treated with tracheal stent before the operation.

\section{Results}

Using these 12 cases, we have verified that $\mathrm{CAD}$ and $3 \mathrm{D}$ visualization techniques can improve surgical precision in patients with thyroid cancer. For all of the cases, the CAD and 3D visualization images were suitable for the detection of the tumor, which was confirmed during the operation. It is difficult to estimate the operation time saved with this method, but the accurate preoperative evaluation is highly beneficial to surgical progress and accuracy and the planning of functional reconstruction. The postoperative histopathology reports are summarized in Table 1 . None of the 12 patients died during the perioperative period. Eight cases underwent radioactive iodine therapy following the operation, and two cases received external radiotherapy. All patients remain alive at the time of this retrospective study.

\section{Case Presentations Case I}

A 74-year-old woman was admitted to the hospital with a recurrent neck mass, hoarseness, and chronic dyspnea for four months. During the past 14 years, the patient had undergone four thyroid surgeries, yet the postoperative pathology was unknown. The neck mass was diagnosed as papillary thyroid carcinoma by ultrasound-guided biopsy cytology. The electronic laryngoscope suggested bilateral vocal cord paralysis and smooth throat. Preoperative neck and enhanced chest $\mathrm{CT}$ showed that the soft tissue in the thyroid region was irregularly thickened. The right side of the neck had a large lesion of $3.1 \times 2.5 \times 4.5 \mathrm{~cm}$. The lesion invaded the right parapharyngeal space and right flank of the thyroid cartilage. There were several small nodules in both lungs, which were determined to be lung metastases. The patient was diagnosed with recurrent T4N1bM1 papillary thyroid carcinoma.

Based on CT images, it was unclear whether the laryngeal function could be preserved. Next, CAD was used to evaluate the tumor before the operation. CAD images revealed that the tumor invaded the right side of the thyroid cartilage and the hyoid bone, yet the laryngeal cavity remained intact. The preoperative surgical plan included a complete thyroidectomy, bilateral neck and central lymph node dissection, partial laryngectomy, partial hyoid osteotomy, and tracheostomy. The extent of tumor involvement observed during the operation was consistent with the preoperative $\mathrm{CAD}$ assessment (Figure 1). The postoperative diagnosis was recurrent and metastatic papillary thyroid carcinoma with poorly differentiated thyroid carcinoma components. The multidisciplinary oncology team recommended supplementation with radiochemotherapy after surgery, yet the patient refused this treatment. The patient showed no signs of tumor recurrence within four months of the operation, and there was no progression of the lung metastases.

\section{Case 2}

A 46-year-old woman presented with a mass in the neck that had increased in size for the past five years. The patient also had chronic hoarseness for the previous two

Table I Demographics and Clinical Data of the 12 Cases Retrospectively Analyzed in This Study

\begin{tabular}{|c|c|c|c|c|c|c|}
\hline Patient & $\begin{array}{l}\text { Age } \\
\text { (Years) }\end{array}$ & Sex & Pathology & TNM & Dyspnea & Anatomy Invaded \\
\hline I & 46 & $\mathrm{~F}$ & Substernal goiter & - & - & - \\
\hline 2 & 47 & M & $\begin{array}{l}\text { PTC with Langerhans } \\
\text { cell histiocytosis }\end{array}$ & T4bNIbM0 & III & Trachea, right common carotid artery, right internal jugular vein \\
\hline 3 & 69 & $\mathrm{~F}$ & Substernal goiter & - & - & - \\
\hline 4 & 74 & $\mathrm{~F}$ & PTC with PDTC & T4aNIbMI & III & Thyroid cartilage and the hyoid bone \\
\hline 5 & 61 & $\mathrm{~F}$ & PTC with SCC & $\mathrm{T} 4 \mathrm{aNIbMO}$ & III & Trachea, right common carotid artery, brachiocephalic trunk \\
\hline 6 & 44 & $\mathrm{~F}$ & MTC & - & - & Brachiocephalic vein \\
\hline 7 & 60 & $\mathrm{~F}$ & PDTC & $\mathrm{T} 4 \mathrm{aNI} \mathrm{bMO}$ & III & Trachea (5 rings) \\
\hline 8 & 61 & $\mathrm{~F}$ & PTC with SCC & T4bNIbMI & III & $\begin{array}{l}\text { Trachea, right internal jugular vein, right subclavian artery, right } \\
\text { recurrent laryngeal nerve, and vagus nerve }\end{array}$ \\
\hline 9 & 55 & M & PTC & $\mathrm{T} 4 \mathrm{aNI} \mathrm{bMO}$ & II & Trachea (4 rings) \\
\hline 10 & 49 & M & Recurrent PTC & T4aNIbM0 & II & Trachea (4 rings) \\
\hline 11 & 68 & $\mathrm{~F}$ & Recurrent PTC & T4aNIbM0 & II & Trachea ( 3 rings), right recurrent laryngeal nerve, and vagus nerve \\
\hline 12 & 51 & M & PTC & T3bNIbM0 & - & Belt-shaped muscle, constrictor muscle of the pharynx \\
\hline
\end{tabular}

Abbreviations: PTC, papillary thyroid carcinoma; SCC, squamous cell carcinoma; MTC, medullary thyroid carcinoma; PDTC, poorly differentiated thyroid carcinoma. 
A

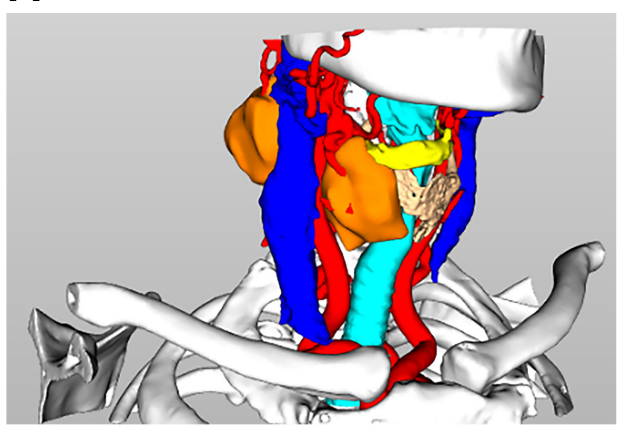

C

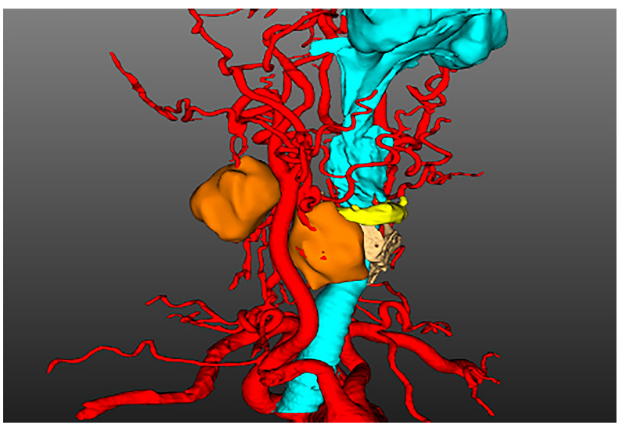

B

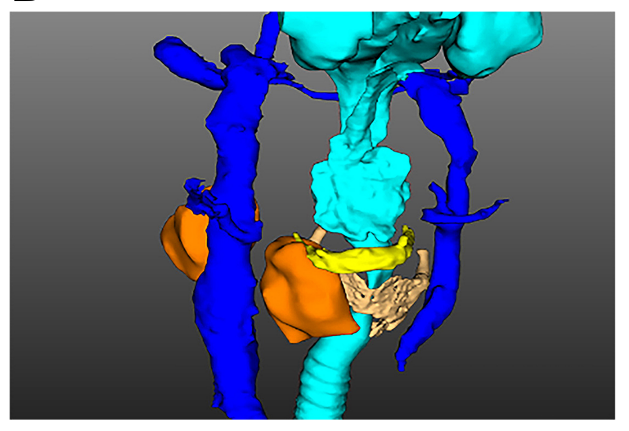

D

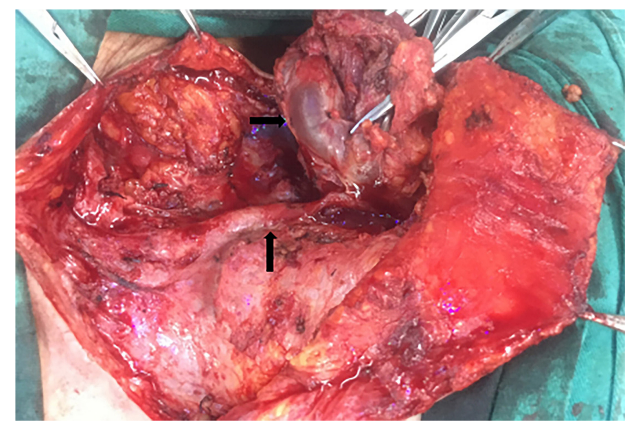

E

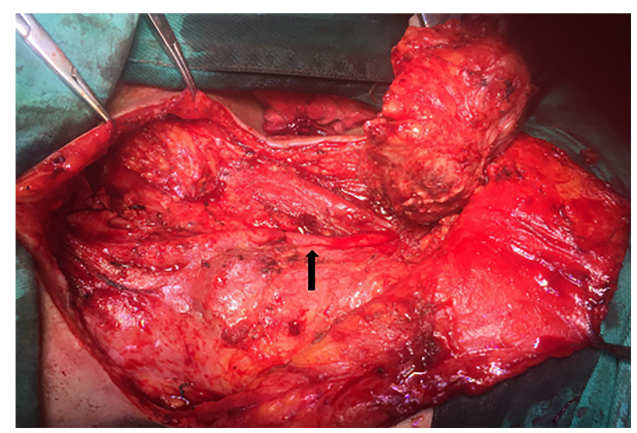

Figure I (A-C) Three-dimensional (3D) reconstruction images revealed that the tumor invaded into the right internal jugular vein and the right side of the thyroid cartilage and hyoid bone. However, the tumor did not adhere to the right common carotid artery. (D and $\mathbf{E})$ Tumor was found to invade the right internal jugular vein during surgery. The vein was removed, while the right common carotid artery was protected.

years. CT imaging revealed a large soft tissue mass (approximately $12 \times 6 \times 5 \mathrm{~cm}$ ) in the right lobe of the thyroid gland. The lower boundary of the tumor was located below the plane of the aortic arch. Color Doppler ultrasound showed multiple nodules on the right side of the thyroid gland that was growing towards the back of the sternum. Based on CT findings, sternotomy was considered the first choice of treatment. Next, the tumors were assessed with CAD visualization techniques.

The 3D CAD images revealed that the tumor was multinodular and extended deep into the superior mediastinum (Figure 2A-C). However, the boundaries of the tumor were evident from the CAD images. There was a gap between the tumor and surrounding organs. The recommended surgical plan included a subtotal thyroidectomy using the neck approach. During the operation, surgeons discovered that multiple nodules fused into a lump of about $13 \times 7 \times 6 \mathrm{~cm}$, and the lower portion of the tumor $(8 \times 6 \times 5 \mathrm{~cm})$ extended into the upper mediastinum (Figure 2D and E). The lower boundary was found to be lower than the aortic arch. The operation was successful, and the patient was discharged five days postsurgery. The postoperative diagnosis was substernal goiter.

\section{Discussion}

\section{Characteristics of Refractory Thyroid} Tumors

Surgery is considered the gold standard for the treatment of both benign and malignant thyroid nodules. The prognosis of thyroid cancer is good for most cases, yet invasion 
A

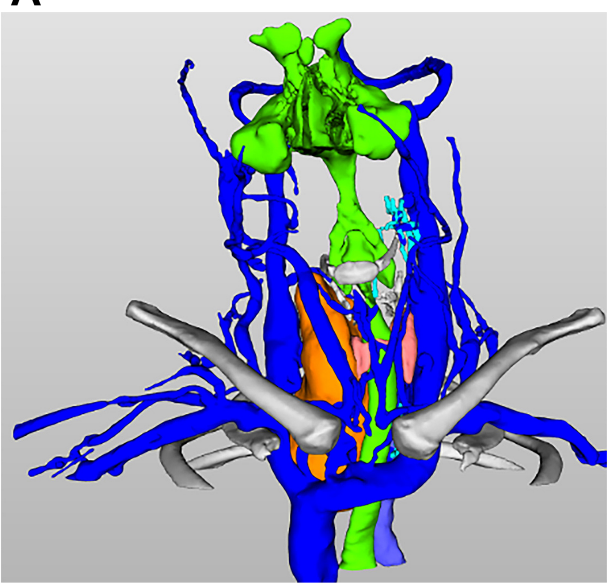

C

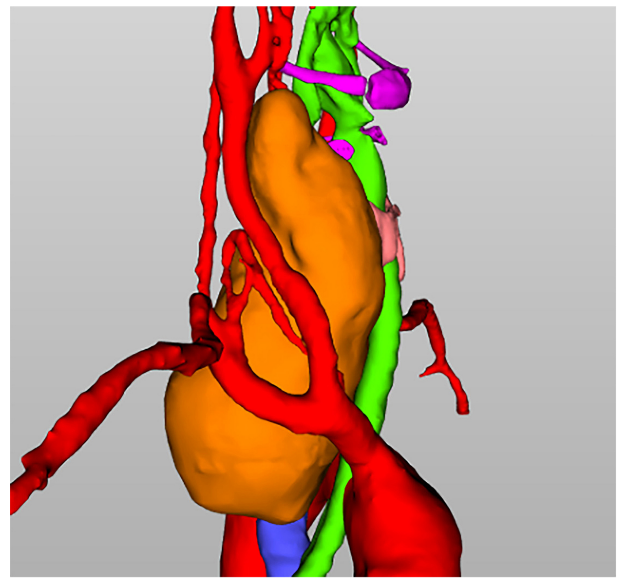

B

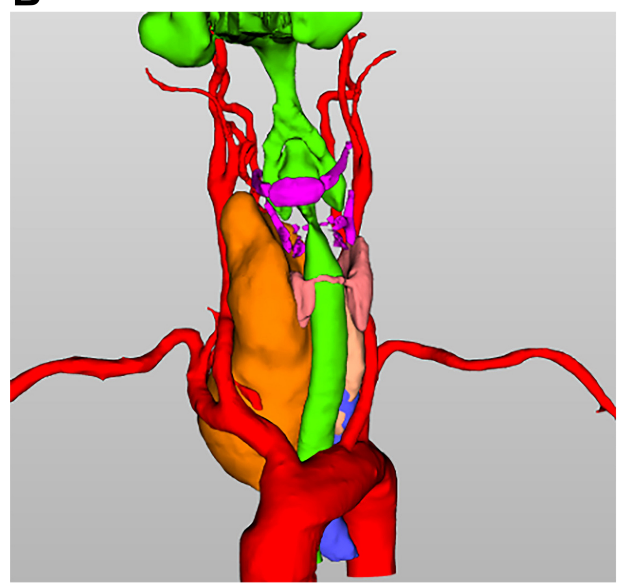

D

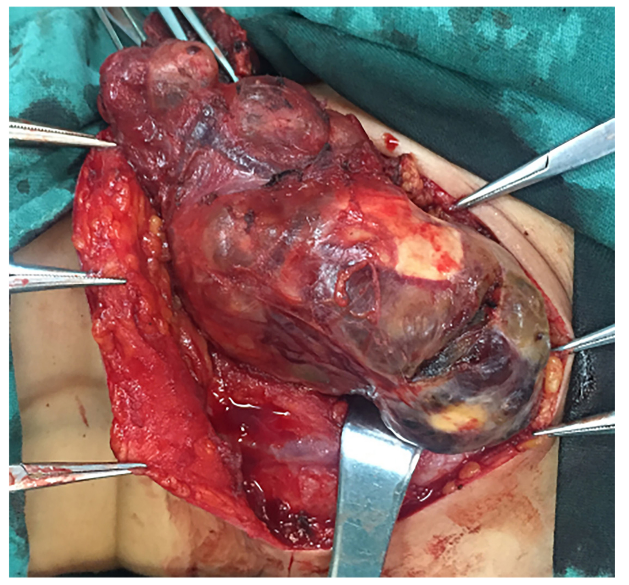

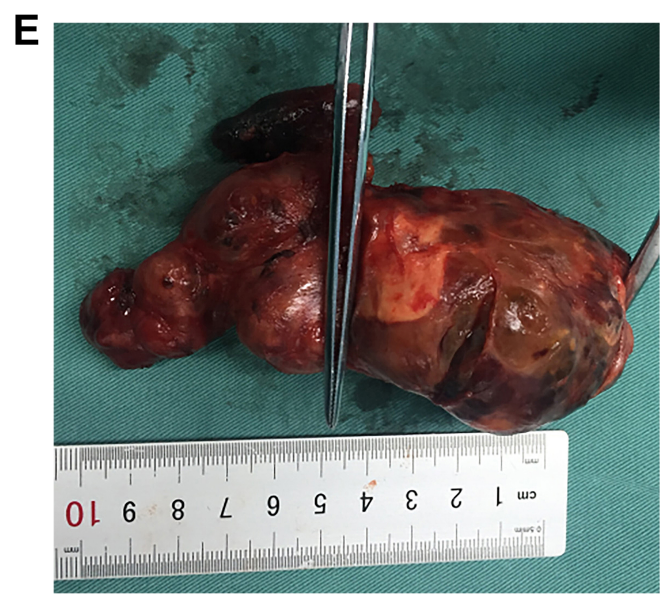

Figure 2 (A-C) Tumor was found to extend deep into the upper mediastinum by CAD and 3D visualization. However, the tumor boundary was found to be distinct. (D and E) Using the imaging data for guidance, the entire tumor was excised successfully through a neck incision.

of the larynx or trachea can diminish the prognosis and complicate the treatment of this disease. In addition, the tumors may cause airway obstructions, hemorrhages, and dyspnea, which are important causes of thyroid cancer death. Locally advanced thyroid cancer can invade the blood vessels of the neck or chest, such as the carotid artery, internal jugular vein, brachiocephalic trunk, and superior vena cava. While radical resection of the primary tumor and invaded organs can improve the overall survival of patients, the operation often required complex respiratory, digestive, and vascular reconstruction in some cases. While a large goiter or adenoma may be benign, the 
compression of the upper respiratory tract may also lead to life-threatening conditions requiring urgent intervention. Patients requiring complicated thyroid surgeries often have postoperative complications due to the involvement of the larynx, trachea, neck or mediastinal vessels. Hence, the accurate and timely preoperative assessment of tumor invasion is of critical importance for determining the best course of treatment.

\section{Current Status of CAD and 3D Visualization Technology}

The $3 \mathrm{D}$ visualization of the surgical area can be accomplished with the aid of a computer, which is commonly used in industry and medicine. The CAD and $3 \mathrm{D}$ visualization technology transform the previous "empirical thinking" into "quantitative thinking." The application of CAD generally includes four steps, namely, data acquisition, 3D reconstruction, surgical planning, and postoperative evaluation. ${ }^{8}$ On this basis, the operation can also be assisted by computer-aided manufacturing, 3D printing, intraoperative navigation, among other things. Compared with traditional surgery, CAD has the following advantages: it facilitates preoperative communication, improves the accuracy of surgery, shortens the operation time, and improves the protection and recovery of postoperative function. At present, CAD also has some defects, such as the long preparation time before surgery and insufficient information for the judgment of tumor edges. ${ }^{8-10}$

\section{Application of CAD and 3D Visualization Technologies for Refractory Thyroid}

\section{Tumors}

For complex thyroid tumors, the CAD technology can clearly and stereoscopically display the size, location, and the extent of tumor invasion. In addition, it allows for visualization of the connections between the tumor and important blood vessels or organs. It can also play an essential role in determining the range of tumors and the primary source of the tumor. CAD has unique advantages in the diagnosis and differential diagnosis of cancer.

Advanced thyroid cancer frequently invades surrounding organs and tissues, most commonly the larynx or trachea. Radical resection of the tumors and affected organs results in better overall survival, while small tumor residuals or metastases increase the risk of recurrence and diminish the overall survival of patients. ${ }^{11,12}$ In our clinical experience, radical resection is the key to improving the survival of patients with differentiated thyroid cancer, even when the surgical margin is small. ${ }^{13}$ Therefore, preoperative evaluation of the extent of tracheal invasion is particularly crucial as the degree of invasion determines the extent of surgical resection and the manner of reconstruction. It has been reported that end-to-end anastomosis can be done within $5-6 \mathrm{~cm}$ of the trachea. More extended tracheal defects may reduce the tension only by relaxing the lower lung ligament, cutting off the left main bronchus, and releasing the thyrohyoid membrane to drop the larynx. However, the risk of surgical complications increases significantly in these cases. ${ }^{14-16}$

The CAD visualization technology can intuitively provide surgeons with adequate data to calculate the length and circumference of the airway invasion, which will help to accurately determine the scope of surgery and reconstruction plans before the operation. In return, this reduces the blindness of surgery and increases the positive surgical outcomes. For patients with large tumors that wrap around the common carotid artery, the relationship between the tumor and blood vessels can be evaluated by CAD visualization technique before surgery, which allows for necessary preparation before surgery. ${ }^{17}$ For thyroid cancer with invasion of the upper mediastinum, preoperative assessment of the tumor extent is essential for determining the optimal surgical approach. However, it is important to note that complex 3D imaging is not a replacement for careful endoscopy, as it lacks the ability to effectively visualize mucosal changes.

A large goiter that compresses the trachea requires immediate surgical intervention. While the lesion is benign, the surgical procedure is both complicated and dangerous. The preoperative evaluation of the tumor's location and its relationship with the trachea, esophagus, and blood vessels of the neck and chest are of critical importance. ${ }^{18}$ CAD visualization technology may also allow for preoperative anesthesia risk assessment and the development of an intubation program in case of an emergency. In this group, two cases presented with benign lesions. After preoperative CAD evaluation, the tumor was located above the chest's large blood vessels with an apparent gap between the tumor and blood vessels of the neck and chest. Therefore, the preoperative evaluation does not require a sternotomy. Combined with electronic bronchoscopy, CAD, and CT images, the two patients were successfully intubated. If the airway is severely compressed, the risk of anesthetic intubation may be reduced by preoperative airway stent implantation. ${ }^{19}$ 


\section{Conclusions}

CAD images can provide essential 3D information that can assist physicians in the diagnosis of cancer, development of surgical plans, optimization of surgical approaches, and evaluation of vascular and airway reconstruction plans. In addition, CAD may aid physicians in multiple disciplines of medicine. For example, experts in anesthesiology and intensive care units can fully evaluate perioperative conditions and promote intraoperative coordination and postoperative supportive care. Oncologists and radiation experts can assess the extent of the tumor and the intraoperative situation, allowing for the development of personalized treatment plans post-surgery. The CAD technology may also help physicians-in-training learn new surgical procedures and enable repeated trials and errors. Due to the simplicity of the images, physicians can use the 3D images to help explain the condition and surgical plan to patients and their families, which is conducive to effective communication between the physicians and patients regarding the possible surgical risks, postoperative complications, and postoperative disabilities. Therefore, the CAD technology can play an important role in the diagnosis and treatment of thyroid surgery.

\section{Author Contributions}

All authors made substantial contributions to the conception and design, acquisition of data, or analysis and interpretation of data. In addition, all authors took part in drafting the article or revising it for important intellectual content, gave final approval of the version to be published, and agree to be held accountable for all aspects of the work.

\section{Funding}

This research did not receive any specific grant from funding agencies in the public, commercial, or not-forprofit sectors.

\section{Disclosure}

The authors have declared that no competing interests exist.

\section{References}

1. Sun JW, Xu XJ, Cai QM. Epidemiological study on thyroid cancer in China. China Cancer. 2013;22:690-693.
2. Bray F, Ferlay J, Soerjomataram I, et al. Global cancer statistics 2018: GLOBOCAN estimates of incidence and mortality worldwide for 36 cancers in 185 countries. CA Cancer J Clin. 2018;68 (6):394-424. doi:10.3322/caac.21492

3. Haugen BR, Alexander EK, Bible KC, et al. 2015 American Thyroid Association management guidelines for adult patients with thyroid nodules and differentiated thyroid cancer: the American Thyroid Association guidelines task force on thyroid nodules and differentiated thyroid cancer. Thyroid. 2016;26(1):1-133. doi:10.1089/ thy. 2015.0020

4. Wang LY, Nixon IJ, Patel SG, et al. Operative management of locally advanced, differentiated thyroid cancer. Surgery. 2016;160 (3):738-746. doi:10.1016/j.surg.2016.04.027

5. Sun J. Application of computer aided surgery technique in oral and maxillofacial surgery. Chin J Prac Stom. 2014;7:329-334.

6. Rana M, Essig H, Eckardt AM, et al. Advances and innovations in computer-assisted head and neck oncologic surgery. J Craniofac Surg. 2012;23(1):272-278. doi:10.1097/SCS.0b013e318241bac7

7. Shin D-H, Mark EJ, Suen HC, et al. Pathologic staging of papillary carcinoma of the thyroid with airway invasion based on the anatomic manner of extension to the trachea: a clinicopathologic study based on 22 patients who underwent thyroidectomy and airway resection. Hum Pathol. 1993;24(8):866-870. doi:10.1016/ 0046-8177(93)90136-5

8. Han HH, Kim HY, Lee JY. The pros and cons of computer-aided surgery for segmental mandibular reconstruction after oncological surgery. Arch Craniofac Surg. 2017;18(3):149-154. doi:10.7181/ acfs.2017.18.3.149

9. De Maesschalck T, Courvoisier DS, Scolozzi P. Computer-assisted versus traditional freehand technique in fibular free flap mandibular reconstruction: a morphological comparative study. Eur Arch Otorhinolaryngol. 2017;274(1):517-526. doi:10.1007/s00405-0164246-4

10. Berrone M, Crosetti E, Succo G. Repositioning template for mandibular reconstruction with fibular free flaps: an alternative technique to pre-plating and virtual surgical planning. Acta Otorhinolaryngol Ital. 2014;34(4):278-282.

11. Dedivitis RA, Guimarães AV. Locally invasive papillary thyroid carcinoma. Braz J Otorhinolaryngol. 2002;68:687-691.

12. Mossetti C, Palestini N, Bruna MC, et al. Segmental tracheal resection for invasive differentiated thyroid carcinoma. Our experience in eight cases. Langenbecks Arch Surg. 2013;398(8):1075-1082. doi:10.1007/s00423-013-1127-9

13. Wang ZH, Cai YC, Li CH. Surgical treatment of differentiated thyroid carcinoma with larynx and trachea invasion. Chin J Endocr Surg. 2014;8:278-281.

14. Wang HD, Yang K, Jing T. Surgical management of primary tracheobronchial tumor. Acta Acad Med Mil Tert. 2005;27:2444-2446.

15. Auchincloss HG, Wright CD. Complications after tracheal resection and reconstruction: prevention and treatment. $J$ Thorac Dis. 2016;8 (Suppl 2):S160-167. doi:10.3978/j.issn.2072-1439.2016.01.86

16. Meyer R. U.K. Kesselring, Reconstructive Surgery of the Trachea. New York: Thieme-Stratton; 1982:136.

17. Zhang ZY, Zhu HG, Sun J, et al. Surgery of the carotid artery: a retrospective study of 35 consecutive cases. Chinese J Stomatology. 2006;41(1):25-28.

18. He L, Wang J, Wang ZW, et al. Experience in treating giant substernal goiter through combined cervicothoracic approach. $J$ Clin Surg. 2014;556-558.

19. Zhang YJ, Wang ZH, Bao Y. Clinical application of tracheal stent in surgical operation for tracheostenosis caused by thyroid tumor. Chin $J$ Endocr Surg. 2018;12:47-50. 


\section{Publish your work in this journal}

Cancer Management and Research is an international, peer-reviewed open access journal focusing on cancer research and the optimal use of preventative and integrated treatment interventions to achieve improved outcomes, enhanced survival and quality of life for the cancer patient.
The manuscript management system is completely online and includes a very quick and fair peer-review system, which is all easy to use. Visit http://www.dovepress.com/testimonials.php to read real quotes from published authors.

Submit your manuscript here: https://www.dovepress.com/cancer-management-and-research-journal 